\title{
Análise crítica da estimativa do número de portadores de esquistossomose mansoni no Brasil
}

\author{
Critical analysis of the estimated number of schistosomiasis \\ mansoni carriers in Brazil
}

\author{
Naftale Katz e Sérgio Viana Peixoto
}

\begin{abstract}
Resumo Estimou-se o número de portadores da infecção por Schistosoma mansoni no Brasil baseando-se no resultado de exames parasitológicos de fezes realizados pela Fundação Nacional de Saúde nos anos de 1996 e 1997 e os dados de população de 18 estados da federação no levantamento do IBGE. Os dados permitiram estimar em 7,1 milhões de portadores de esquistossomose em 1996 e em 6,3 milhões em 1997. Esses números podem não representar a realidade, pois a amostra da população examinada não foi selecionada visando este objetivo. A ausência de dados precisos indica a necessidade de adequado levantamento nacional da prevalência da esquistossomose que continua a ser importante endemia parasitária, justificando esforços maiores para o seu controle no Brasil.
\end{abstract}

Palavras-chaves: Esquistossomose mansoni. Brasil. Prevalência.

\begin{abstract}
The number of carriers of Schistosoma mansoni infection in Brazil was estimated based on the results of parasitological examinations of feces carried out by the Fundação Nacional de Saúde (FNS - National Health Foundation) in 1996 and 1997, as well as population data from 18 states collected by the Instituto Brasileiro de Geografia e Estatística (IBGE-Brazilian Institute of Geography and Statistics). This information allowed the number of carriers of schistosomiasis mansoni to be estimated at 7.1 million in 1996 and 6.3 million in 1997. These figures may not reflect the true situation since the population sample used was not originally selected for this purpose. The absence of precise data indicates the need for an adequate national survey of the prevalence of schistosomiasis, which continues to be an important endemic parasitic disease, justifying greater efforts for its control in Brazil.
\end{abstract}

Key-words: Schistosomiasis mansoni. Brazil. Prevalence.

A esquistossomose mansoni é endêmica em cinquenta e dois países da América do Sul, do Caribe, da África e da região oriental do Mediterrâneo ${ }^{13}$. Constituindo grande problema de saúde pública, essa endemia está associada, à pobreza e ao baixo desenvolvimento econômico que gera a necessidade de utilização de águas naturais contaminadas para o exercício da agricultura, trabalho doméstico e/ou lazer.
No Brasil, o primeiro inquérito nacional de prevalência da esquistossomose foi realizado pela Divisão de Organização Sanitária publicado por Pellon e Teixeira em $1950^{\circ}$. Esse levantamento foi realizado em escolares de 7 a 14 anos de idade em 11 estados brasileiros, através do exame parasitológico de fezes pela técnica de sedimentação em água ${ }^{2}$. Foram realizados 440.786 exames, obtendo-se prevalência média

Centro de Pesquisas René Rachou/Fundação Oswaldo Cruz (FIOCRUZ), Belo Horizonte, MG, Brasil.

Endereço para correspondência: Dr. Naftale Katz. CPqRR/FIOCRUZ. Av. Augusto de Lima 1715, Barro Preto, 30190-002 Belo Horizonte, MG, Brasil.

Fax 5531 295-3115.

e-mail: nkatz@cpqrr.fiocruz.br

Recebido para publicação em 1/10/99 
de $10,1 \%$ para o Brasil. Os autores estimaram em 2,6 milhões o número total de casos de esquistossomose nos 11 estados pesquisados.

No ano de 1953, foram estudados pelos mesmos autores, outros cinco estados brasileiros, que compreendiam áreas supostamente não endêmicas. Os resultados desse inquérito mostraram $0,08 \%$ de amostras fecais positivas ${ }^{10}$.

A prevalência média da helmintose para o Brasil foi calculada reunindo os dados dos dois estudos, resultando em $7,26 \%$ para os 16 estados pesquisados. Posteriormente, outro grande inquérito foi promovido pelo Ministério da Saúde/ SUCAM entre 1976 e 1981, em 18 unidades da federação. Foram examinadas 333.427 amostras de fezes, de escolares de 7 a 14 anos, pelo método de Kato-Katz ${ }^{6}$, com 3,75\% de exames positivos ${ }^{12}$ (Tabela 1).

Em 1997, um assessor da Organização Mundial da Saúde (OMS) em visita ao Programa

Tabela 1 - Prevalência da esquistossomose observada em dois inquéritos de abrangência nacional, por estado brasileiro em 1950/1953 e 1977/1981.

\begin{tabular}{|c|c|c|}
\hline \multirow[b]{2}{*}{ Estado } & \multicolumn{2}{|c|}{ Prevalência da doença } \\
\hline & $1950 / 53^{*}$ & $1977 / 81^{\text {** }}$ \\
\hline 1. Rondônia & NE & NE \\
\hline 2. Acre & $\mathrm{NE}$ & NE \\
\hline 3. Amazonas & NE & NE \\
\hline 4. Roraima & NE & $\mathrm{NE}$ \\
\hline 5. Pará & NE & 0,40 \\
\hline 6. Amapá & NE & $\mathrm{NE}$ \\
\hline 7. Tocantins & NE & $\mathrm{NE}$ \\
\hline 8. Maranhão & 0,46 & 3,20 \\
\hline 9. Piauí & 0,04 & 0,00 \\
\hline 10. Ceará & 0,94 & 2,90 \\
\hline 11. Rio Grande do Norte & 2,32 & 0,60 \\
\hline 12. Paraíba & 7,49 & 5,20 \\
\hline 13. Pernambuco & 25,09 & 9,30 \\
\hline 14. Alagoas & 19,75 & 21,8 \\
\hline 15. Sergipe & 29,80 & 31,7 \\
\hline 16. Bahia & 16,55 & $\mathrm{NE}$ \\
\hline 17. Minas Gerais & 4,92 & 10,10 \\
\hline 18. Espírito Santo & 1,62 & 2,60 \\
\hline 19. Rio de Janeiro & 0,10 & 0,60 \\
\hline 20. São Paulo & NE & $\mathrm{NE}$ \\
\hline 21. Paraná & 0,12 & 0,40 \\
\hline 22. Santa Catarina & 0,00 & 0,00 \\
\hline 23. Rio Grande do Sul & $\mathrm{NE}$ & $\mathrm{NE}$ \\
\hline 24. Mato Grosso do Sul & $\mathrm{NE}$ & $\mathrm{NE}$ \\
\hline 25. Mato Grosso & 0,01 & 0,90 \\
\hline 26. Goiás & 0,08 & 0,10 \\
\hline 27. Distrito Federal & $\mathrm{NE}$ & 0,70 \\
\hline Brasil & 7,26 & 3,75 \\
\hline
\end{tabular}

* Pellon e Teixeira, 1950 e 1953; " SUCAM, 1981; NE = não examinado.

de Controle da Esquistossomose no Brasil, baseado em dados fornecidos pela Fundação Nacional de Saúde (FNS), cita em seu relatório, a estimativa de que 25 milhões de pessoas estão expostas ao risco de infecção, existindo 2,5 milhões de infectadas. $\mathrm{O}$ autor ressalta o problema da esquistossomose no país, relacionando sua endemicidade com a pobreza e baixo desenvolvimento econômico. Além disso, recomenda a intensificação das atividades educativas e o controle integrado de outras parasitoses, o que beneficiaria o programa nacional de controle ${ }^{1}$. 
Recentemente, Passos e Amaral (1998) ${ }^{8}$, baseados nos mesmos dados da FNS, estimaram o número de portadores de esquistossomose no país em 2,5 milhões e cerca de 25 milhões expostos ao risco de contraí-la. Estes dados também estão repetidos em recente publicação da $\mathrm{OMS}^{14}$. Os autores relataram os percentuais de positividade para esquistossomose nos anos de 1975 a 1997, sendo que na década de 1990, esses valores oscilaram entre 7 e $11 \%$.

Além dos dados de prevalência, outra medida de morbidade a ser considerada é o número de internações e óbitos devido à esquistossomose, divulgados pelo Sistema Único de Saúde (SUS) * para o Brasil. A observação dos dados hospitalares mostram redução do número de internações na década de 1990, passando de 3.293 em 1991 para $1.314 \mathrm{em} 1998$. A mortalidade é ainda bastante expressiva, levando-se em consideração que 4.391 pessoas morreram no Brasil entre $1990 \mathrm{e}$ 1997 devido à doença ${ }^{11}$.

Para a estimativa segura da prevalência da esquistossomose, necessita-se de levantamentos parasitológicos feitos com amostragem adequada, em nível nacional, levando em consideração que a prevalência da parasitose não apresenta distribuição regular. Embora estes não existam, o presente trabalho tem como objetivo estimar o número de portadores de esquistossomose no Brasil, a partir de uma análise crítica dos dados da Fundação Nacional de Saúde (FNS), recentemente publicados. No ano de 1996 e 1997 , foram realizados respectivamente $2.492 .567 \mathrm{e}$ 1.515.723 exames parasitológicos de fezes.

O cálculo do número de indivíduos positivos para esquistossomose foi realizado assumindose que a prevalência da infecção nos 18 estados da federação nos anos de 1996 e 1997, citados por Passos e Amaral $(1998)^{8}$ seria representativa da população dos estados; os dados populacionais foram obtidos da Fundação Instituto Brasileiro de Geografia e Estatística (IBGE) ${ }^{3}$.

As Tabelas 2 e 3 mostram os cálculos realizados para os 18 estados brasileiros. Segundo essas estimativas, o Brasil possuía, aproximadamente, 7,4 milhões de portadores de esquistossomose em 1996 e 8,3 milhões em 1997.

Para a análise dos resultados de 1997, o valor encontrado para o estado de Goiás foi excluído, pois essa prevalência reflete a descoberta de um novo foco da doença. Da mesma forma, os altos percentuais de positividade observados no Distrito Federal são resultado de achados entre migrantes e de buscas dirigidas ${ }^{8}$.

Excluindo-se o número de positivos de Goiás e do Distrito Federal, encontra-se um total de 7.072.661 portadores de esquistossomose em 1996 e de 6.352.678 em 1997.

Deve-se chamar atenção de que estes resultados podem não representar a realidade, pois a prevalência usada para esse cálculo não foi obtida através de amostra representativa da população nos diferentes estados brasileiros. No entanto, esse número é muito diferente dos 2,5 milhões estimados por Passos e Amaral ${ }^{8}$.

Analisando os dados divulgados pela FNS e publicados por Passos e Amaral $(1998)^{8}$, observase que foram examinados $1,6 \%$ da população brasileira em 1996 e 0,9\% em 1997. Como não foi realizada avaliação detalhada das microregiões efetivamente examinadas, esses dados podem estar mascarando a prevalência real da infecção, pois os grupos populacionais estudados podem ser provenientes de regiões endêmicas, indenes ou mesmo abranger regiões com taxas de infecção muito variáveis. Da mesma forma, há o risco de terem sido selecionados, grupos populacionais residentes em regiões onde programas de controle estão alcançando resultados satisfatórios ou mesmo terem sido realizados alguns meses pós-terapêutica, reduzindo, artificialmente, a estimativa de prevalência da infecção no país.

Outro fato a ser mencionado, é o número de indivíduos expostos ao risco de contrair a infecção. A Organização Mundial de Saúde (OMS) estimou que, em 1990, 30 milhões de pessoas, no Brasil, estavam expostas ao risco de infecção $0^{13} \mathrm{e}$ em outra publicação, 25 milhões $^{14}$. Todavia, baseando-se nos dados de 1997, a população total dos 13 estados brasileiros, sabidamente com áreas endêmicas, com prevalência da esquistossomose superior a $4 \%$, era de 70.168 .906 indivíduos. Além disso, o Comitê da OMS destaca o aumento da esquistossomose urbana no nordeste do país e em outras regiões do mundo, mostrando que essa endemia ainda é uma ameaça constante nos países em desenvolvimento. 
Tabela 2 - Estimativa do número de portadores de esquistossomose mansoni, por estado brasileiro em 1996.

\begin{tabular}{|c|c|c|c|}
\hline Estado & População* & Prevalência** & No positivos \\
\hline 1. Rondônia & 1.231 .007 & 5,01 & 61.673 \\
\hline 2. Acre & 483.726 & NE & $N E$ \\
\hline 3. Amazonas & 2.389 .279 & NE & $\mathrm{NE}$ \\
\hline 4. Roraima & 247.131 & NE & NE \\
\hline 5. Pará & 5.510 .849 & 3,22 & 177.449 \\
\hline 6. Amapá & 379.459 & $N E$ & $\mathrm{NE}$ \\
\hline 7. Tocantins & 1.048 .642 & $\mathrm{NE}$ & $\mathrm{NE}$ \\
\hline 8. Maranhão & 5.222 .565 & 3,91 & 204.202 \\
\hline 9. Piauí & 2.673 .176 & 0,00 & 0 \\
\hline 10. Ceará & 6.809 .794 & 2,86 & 194.760 \\
\hline 11. Rio Grande do Norte & 2.558 .660 & 5,63 & 144.053 \\
\hline 12. Paraíba & 3.305 .616 & 10,94 & 361.634 \\
\hline 13. Pernambuco & 7.399 .131 & 18,00 & 1.331 .844 \\
\hline 14. Alagoas & 2.633 .339 & 24,87 & 654.911 \\
\hline 15. Sergipe & 1.624 .175 & 21,40 & 347.573 \\
\hline 16. Bahia & 12.541 .745 & 10,33 & 1.295 .562 \\
\hline 17. Minas Gerais & 16.673 .097 & 8,97 & 1.495 .577 \\
\hline 18. Espírito Santo & 2.802 .707 & 7,20 & 201.794 \\
\hline 19. Rio de Janeiro & 13.406 .379 & 1,08 & 144.789 \\
\hline 20. São Paulo & 34.120 .886 & $\mathrm{NE}$ & NE \\
\hline 21. Paraná & 9.003 .804 & 0,45 & 40517 \\
\hline 22. Santa Catarina & 4.875 .244 & 0,24 & 11.701 \\
\hline 23. Rio Grande do Sul & 9.637 .682 & $\mathrm{NE}$ & NE \\
\hline 24. Mato Grosso do Sul & 1.927 .834 & NE & NE \\
\hline 25. Mato Grosso & 2.235 .832 & $\mathrm{NE}$ & NE \\
\hline 26. Goiás & 4.515 .868 & 8,96 & 404.622 \\
\hline 27. Distrito Federal & 1.821 .946 & 18,49 & 336.878 \\
\hline Brasil & 157.079 .573 & 8,75 & 7.409 .539 \\
\hline
\end{tabular}

* Fundação Instituto Brasileiro de Geografia e Estatística - IBGE, 1996; ** Passos e Amaral, 1998; NE = não examinado.

No Brasil, quase $80 \%$ da população pode ser considerada urbana ${ }^{5 *}$, sendo a transmissão dessa doença reconhecida em importantes áreas, como por exemplo, a área metropolitana de Belo Horizonte ${ }^{7}$, não justificando a exclusão das grandes cidades nos levantamentos epidemiológicos realizados ou a serem feitos. Destaque-se que embora nas grandes cidades ou capitais, as áreas de infecção sejam raras ou inexistentes no centro e nos bairros onde habita a população com maior poder econômico, as periferias destas, onde reside a população de baixa renda, reproduzem as más condições de saneamento que permitem a instalação de novos focos de esquistossomose, como ocorre nas regiões rurais ou nas pequenas comunidades.

As considerações citadas nesse estudo, acerca da prevalência da esquistossomose no Brasil, deixam claro a necessidade da realização de um novo inquérito nacional de prevalência, onde as amostras sejam representativas das populações dos estados brasileiros, o que mostrará a real situação dessa helmintose no país. No entanto, embora não existam dados precisos, as estimativas apresentadas mostram a extensão da esquistossomose no Brasil, justificando a necessidade de esforços cada vez maiores, visando ao controle desta importante endemia parasitária.

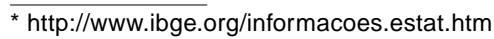


Tabela 3 - Estimativa do número de portadores de esquistossomose mansoni, por estado brasileiro em 1997.

\begin{tabular}{|c|c|c|c|}
\hline Estado & População* & Prevalência** & № positivos \\
\hline 1. Rondônia & 1.255 .522 & 4,31 & 54.113 \\
\hline 2. Acre & 500.185 & NE & NE \\
\hline 3. Amazonas & 2.460 .602 & NE & NE \\
\hline 4. Roraima & 254.499 & $\mathrm{NE}$ & $\mathrm{NE}$ \\
\hline 5. Pará & 5.650 .681 & 3,22 & 181.952 \\
\hline 6. Amapá & 401.916 & $\mathrm{NE}$ & NE \\
\hline 7. Tocantins & 1.080 .753 & NE & NE \\
\hline 8. Maranhão & 5.295 .452 & 6,20 & 328.318 \\
\hline 9. Piauí & 2.695 .876 & 0,00 & 0 \\
\hline 10. Ceará & 6.920 .292 & 4,16 & 287.884 \\
\hline 11. Rio Grande do Norte & 2.594 .340 & 5,62 & 145.802 \\
\hline 12. Paraíba & 3.331 .673 & 8,98 & 299.184 \\
\hline 13. Pernambuco & 7.466 .773 & 17,08 & 1.275 .325 \\
\hline 14. Alagoas & 2.663 .071 & 18,18 & 484.146 \\
\hline 15. Sergipe & 1.657 .164 & 23,60 & 391.091 \\
\hline 16. Bahia & 12.709 .744 & 8,84 & 1.123 .541 \\
\hline 17. Minas Gerais & 16.904 .977 & 7,84 & 1.325 .350 \\
\hline 18. Espírito Santo & 2.853 .098 & 7,09 & 202.285 \\
\hline 19. Rio de Janeiro & 13.555 .657 & 1,14 & 154.534 \\
\hline 20. São Paulo & 34.752 .225 & $\mathrm{NE}$ & NE \\
\hline 21. Paraná & 9.142 .215 & 0,77 & 70.395 \\
\hline 22. Santa Catarina & 4.958 .339 & 0,58 & 28.758 \\
\hline 23. Rio Grande do Sul & 9.762 .110 & NE & NE \\
\hline 24. Mato Grosso do Sul & 1.964 .603 & NE & NE \\
\hline 25. Mato Grosso & 2.287 .846 & $\mathrm{NE}$ & $\mathrm{NE}$ \\
\hline 26. Goiás & 4.639 .785 & 34,61 & 1.605 .830 \\
\hline 27. Distrito Federal & 1.877 .015 & 17,57 & 329.792 \\
\hline Brasil & 159.636 .413 & 9,97 & 8.288 .300 \\
\hline
\end{tabular}

* Fundação Instituto Brasileiro de Geografia e Estatística - IBGE, 1997; ** Passos e Amaral, 1998; NE = Não examinado

\section{REFERÊNCIAS BIBLIOGRÁFICAS}

1. Chitsulo L. Brazil. The Schistosomiasis Control Project (PCE) in Brasilia, the Federal District and the states of Minas Gerais, Paraiba and Alagoas. Travel Report Summary. World Health Organization, 1997.

2. Hoffman WA, Pons JA, Janer JL. The sedimentationconcentration method in schistosomiasis mansoni. The Puerto Rico Journal of Public Health and Tropical Medicine 9:283-291, 1934.

3. Instituto Brasileiro de Geografia e Estatística. Contagem de população 1996, (mimeo)/IBGE, 1996.

4. Instituto Brasileiro de Geografia e Estatística. Estimativa de população 1997, (mimeo)/IBGE, 1997.

5. Instituto Brasileiro de Geografia e Estatística. Indicadores sociais mínimos - Taxa de urbanização. Soliciatada em 09.08.1999. On line. Disponível na Internet <http:// www.ibge.org/informacoes.estat.htm>, 1999.
6. Katz N, Chaves A, Pellegrino J. A simple device for quantitative stool thick-smear technique in schistosomiasis mansoni. Revista do Instituto de Medicina Tropical de São Paulo 14:397-402, 1972.

7. Katz N, Motta E, Oliveira VB, Carvalho EF. Prevalência da esquistossomose em escolares no Estado de Minas Gerais. In: Resumos do XIV Congresso da Sociedade Brasileira de Medicina Tropical, João Pessoa, p. 102, 1978.

8. Passos ADC, Amaral RS. Esquistossomose mansônica: aspectos epidemiológicos e de controle. Revista da Sociedade Brasileira de Medicina Tropical 31 (Suplemento II): 61-74, 1998.

9. Pellon $A B$, Teixeira I. Distribuição geográfica da esquistossomose mansônica no Brasil. Ministério da Educação e Saúde, Departamento Nacional de Saúde, Divisão de Organização Sanitária. In: Oitavo Congresso Brasileiro de Higiene. Rio de Janeiro, 1950. 
10. Pellon $A B$, Teixeira I. O Inquérito Helmintológico Escolar em cinco estados das regiões Leste, Sul e Centro-Oeste. Ministério da Educação e Saúde, Departamento Nacional de Saúde, Divisão de Organização Sanitária. In: XI Congresso Brasileiro de Higiene. Curitiba, 1953.

11. Sistema Único de Saúde. Morbidade Hospitalar do SUS e Mortalidade. Internações e óbitos por Esquistossomíase. Solicitada em 18.08.1999. On line. Disponível na Internet $<$ http://www.datasus.gov.br/cgi/>, 1999.

12. Superintendência de Campanhas de Saúde Pública (SUCAM). Estudo Nacional da Prevalência da
Esquistossomose: 1977 a 1981. Ministério da Saúde (mimeo), 1981.

13. World Health Organization. The control of schistosomiasis. WHO Technical Report Series № 830, Geneve, 1993.

14. World Health Organization. Report of the WHO Informal Consultation on monitoring of drug efficacy in the control of schistosomiasis and intestinal nematodes. Geneve, 10 July, 1998. 